\title{
IS THE GLASS STILL HALF FULL? CAN SOCIAL MARKETING THREAT APPEALS STILL HAVE A ROLE IN REDUCING BINGE DRINKING AMONG FEMALE COLLEGE STUDENTS?
}

\author{
Fergus Murphy, Cork Institute of Technology, Ireland \\ Maurice Murphy, Cork Institute of Technology, Ireland
}

\begin{abstract}
Ireland has one of the highest levels of alcohol consumption in the EU, consuming 10.6 litres of pure alcohol per person in 2003, and increasing to 13.4 litres in 2006. This rise in consumption has led to increases in alcohol-related harm and disease, and has resulted in more than 1,775 deaths according to the Health Research Board (Mongan, et al. 2007). In general, increases in overall consumption are accompanied by a greater incidence of health and social problems. In addition to the high volume of alcohol consumed by people in Ireland, drinking occasions appear to be strongly related to heavy episodic or "binge" drinking patterns. Binge drinking is defined as drinking five or more drinks in a row for men and four or more drinks in a row for women, at least once in the previous two weeks (Dantzer, et al. 2006). The prevalence of alcohol use and associated problems is higher in college populations than in the general public (Evans and Dunn, 1995). In fact, binge drinking has been identified as the number one substance abuse problem in university life (Syre, et al. 1997). A recent international study of drinking among university students in 21 countries found that Ireland had the highest proportions of male and female heavy or binge drinkers (Dantzer, et al. 2006). Women are at an increased risk from alcohol use because they need less alcohol per kilogram of body weight than men to attain the same peak blood alcohol level and level of impairment (Rohsenow, 1998). In their recent report, The Health Research Board (2007) in Ireland state that "if current trends continue, we will see significantly higher numbers of middle-aged women experiencing alcohol-related morbidity or greater premature mortality".
\end{abstract}

This study focuses on Irish female university students and their attitudes towards binge drinking, public service announcements and a harm reduction approach. Its main question is - how can social marketing advertising best reduce the amount of binge drinking among university female students? The research objectives are as follows: (1) How do university female students binge drink? (2) How do they respond in terms of coping strategies to binge drinking? (3) Are social threat appeals more powerful than physical threat appeals in preventing binge drinking among female university students? Five focus groups were conducted between March and April 2009, involving female students at a university in Cork, Ireland. All participants were young female college students aged between 18 and 24 years of age, recruited using a convenience sample. Having completed a discussion on their drinking history, participants were then shown six physical fear advertisements and five social fear advertisements. Reactions to each advertisement were gauged after they were shown. Two leading public health experts in the area of alcohol-related harm in Ireland were also interviewed in April and May 2009. Both individuals were chosen on the basis that they had extensive knowledge of the health effects that binge drinking was causing the Irish public and also on the basis that their own personal opinions and views would be very insightful in gaining an overall view of how serious the public health community viewed binge drinking, especially amongst females. Data was analysed for themes running through both the interviews and the focus groups.

Results show that Ireland has a culture that is immersed and revolves around the consumption of alcohol and this has led to a substantial increase in alcohol related harm in our society. While attending college, this normative behaviour of consuming substantial or excessive amounts of alcohol becomes more regular and sustained. Respondents view their current alcohol consumption as a rite of passage to adulthood and that it will lessen once they attain more responsibilities in adult life. They underestimate the long-term damage that is being done to their health and over-estimate the body's capability to withstand the effects of alcohol. There is an over-reliance on friends to take care of them when drunk as a coping mechanism and therefore a consequent under-estimation of how drunk they actually are. Social marketing messages need to contain images and statistics that will result in shock and fear among the target group of female college students. The advertisements which proved most effective among the participants proved to be physical threat appeals which dealt with the serious issues of sexual assault and rape. Every advertisement shown to the participants dealing with these issues resulted in fear. Sexual assault was never considered a serious threat by students until it was shown in the advertisements. Social marketers should also be aware of the maladaptive coping response of "I would never get like that" or "my friend would take care of me" and devise advertisements that negate this coping response. Therefore, raising awareness about how to drink more safely rather than insisting on abstinence may be a better strategy for health promotion bodies to adopt.

References available upon request 